\title{
LASER AND MULTI-IMAGE REVERSE ENGINEERING SYSTEMS FOR ACCURATE 3D MODELLING OF COMPLEX CULTURAL ARTEFACTS
}

\author{
Ch. Ioannidis, G. Piniotis, S. Soile, F. Bourexis, A.-M. Boutsi, R. Chliverou, M. Tsakiri*
}

School of Rural and Surveying Engineering, National Technical University of Athens, Greece

KEY WORDS: 3D model, laser tracker, close range photogrammetry, reverse engineering

\begin{abstract}
:
The recent scientific and technical developments of reverse engineering methods and tools have broadened the possibilities of applications in the field of cultural heritage conservation. In this paper, two different non-contact reverse engineering systems were utilized for 3D data acquisition of a cultural heritage artefact. The object of interest is a 17th century wooden engraved ecclesiastical sanctuary ciborium. The requirement of the 3D model is to aid the art conservators for the preservation of the wooden material and the restoration of small damages and cracks in the engraved parts, thus requiring accuracy of the model in the order of submillimetre. In this work, a Faro Vantage laser tracker was employed along with the FARO Edge Arm. In addition, image-based modelling was also implemented with a large number of overlapping images acquired with a Canon EOS 6D camera and processed using the well-known Structure from Motion (SfM) method with an auto-calibration procedure. The digital data acquisition and processing procedures of the scanned geometry are described and compared to evaluate the performance of both systems in terms of data acquisition time, processing time, reconstruction precision and final model quality. Whilst models produced with laser scanning and image-based techniques is not a novel approach, the combination of laser tracking and photogrammetric data still presents limited documentation in the field of cultural artefact documentation mainly due to the extremely high cost of the laser tracking systems.
\end{abstract}

\section{INTRODUCTION}

Digital technologies as applied to relic conservation has become a common research topic within the field of cultural heritage preservation. Both the inheritance of traditional techniques and the introduction of advanced technologies rely on the users and their awareness and understanding of cultural heritage. It is important to note that the implemented processes often combine technology and art, and there are always new methods appearing in the technological development. Digital technology is currently one of all these strategies that inherits cultural aspects, improves potency and raises quality. Whilst every technology has advantages and limitations, it is necessary to develop the benefits and avoiding the weaknesses and coming up with possible and effective solutions.

One of the most accurate digital technologies is employed in reverse engineering (RE). RE is the process of analysing an object by deconstructing it to reveal its designs, architecture or to extract knowledge from it, normally with the intention of constructing a new object of similar or extended functionality. The deconstruction is achieved in a digital sense by creating a digital replica using very high accuracy $3 \mathrm{D}$ scanning devices.

$\mathrm{RE}$ is broadly utilized within various applications. For example, in manufacturing (from analysis of an object to quality control of industrial parts), in industrial design (creation of models for virtual reality environments) and technical, industrial and cultural heritage conservation, renovation, maintenance and repair (e.g. Segreto et al., 2016; Bernard et al. 2007; Raja and Fernandes 2008).

The development of RE methods has allowed for interesting applications in the conservation of tangible cultural heritage artefacts. One of the useful and modern applications is that of creating a model for repair needs. RE techniques provide a precious technological solution capable of acquiring the shape and the advent of artefacts with great precision. Recent advances in computer recourses and graphics capability have expanded the visibility of possible damages in digital generated cultural artefacts and RE is further used for inspection and monitoring i.e. detection of artefact changes over the years (e.g. Segreto et al., 2011).

\subsection{Related work}

The first step of the RE procedure is the digital data acquisition. This is a critical stage of the RE procedure and clearly, the choice of the RE data acquisition method affects the quality of the acquired point cloud and, consequently, the resulting reconstructed surface or CAD model. There are many different methods for acquiring shape data, which use diverse mechanisms or phenomena to interact with the surface or volume of the object of interest. The main distinction is between contact methods, that make use of devices such as mechanical touch probes, and non-contact methods, commonly based on light, sound or magnetic fields. Probably, the broadest and most popular methods for geometrical data acquisition are the

\footnotetext{
* Corresponding author
} 
optical-based ones that offer reasonably fast acquisition rates and precision. These are mainly the laser scanning systems and the image-based techniques. Several experiences proposing RE systems by non-contact and non-destructive techniques are documented in the literature (e.g. Pruit et al., 2017).

In this work, the use of laser tracking technique is employed. Laser trackers are widely used for metrology and precision surveys. Depending on the approach, range and instrument itself, the measurement accuracy can vary from millimetre to micron. The laser tracker is often accompanied by special measuring arms and much of the success of this equipment is tied to the laser tracker's ability to measure with seven degrees of freedom (DoF). In fact, there are six DoF that describe the freedom of movement of a rigid body in 3D space and the $7^{\text {th }}$ DoF refers to the move through a larger work envelope (Martin, 2017). A laser tracker can gather information in three different ways: by following a small- mirrored sphere, by tracking a wireless, armless contact probe, or by tracking a handheld scanner. The operator should choose the most appropriate data acquisition method, or combination of tracking tools, for the job at hand.

The most attractive alternative for data acquisition in RE is photogrammetric image-based 3D modelling techniques which have the major advantage of being low-cost, portable, flexible and able to deliver highly detailed geometries and textures. Objects with amorphous geometries, structured surfaces, many edges, many corresponding image points and an inhomogeneous colouring are best suited for this technique. Objects that produce rather bad or no results have unstructured, monochrome, translucent, reflective, and/or self-resembling surfaces and most likely, cultural heritage artefacts fall in this category thus, being problematic for image-based 3D modelling (Nikolae et al., 2014; Schaich, 2013).

\subsection{Aim of work}

Nowadays, the combination of both photogrammetric and range data (structured light, coded light or laser light) techniques are used for $3 \mathrm{D}$ modelling when applied to cultural heritage to enhance qualities of each one and achieve greater results (e.g. Clini et al. 2016; Galo et al., 2013). There is however, limited documentation on the use of RE laser tracking systems combined with photogrammetric techniques on the documentation of cultural artefacts. The aim of this paper is therefore, to describe the rigorous workflow of the RE 3D acquisition and modelling with non-invasive, high density and of extremely high accuracy techniques for a case study of cultural heritage artefact for conservation purposes. The object of interest is a 17th century wooden engraved ecclesiastical sanctuary ciborium of dimensions $1.40 \mathrm{~m} \times 1.40 \mathrm{~m} \times 3.00 \mathrm{~m}$ (Fig. 1). The ciborium is a portable altar that is supported on four columns/legs and is placed in the naves of Greek churches. It is referred to as the 'Holy Table' and in Greek Orthodox churches is always located in the centre of the altar. The specific ciborium is situated in the church of Agios Stefanos in Meteora, Greece and is currently used for the daily ecclesiastical services of the monastery. The wooden material is exposed uninterruptedly in localised temperatures due to the use of candles and scents. In addition, the wood has been treated for woodworms and currently it presents small cracks or missing parts and it is within the priorities of the monastery to restore it.

Due to the complexity of this object, the most significant part of the paper refers to overcoming the difficulties of acquisition and the reconstruction of the texture. The remainder of the paper is outlined as follows. In Section 2, the laser tracking pipeline is described where the data acquisition methodology is presented including a detailed description of the procedure (section 2.1). Section 2.2 provides the data processing steps that have been followed and the results. In Section 3, the digital image data acquisition is described (section 3.1) and section 3.2 presents the results of the processing steps. Section 4 provides a comparison between the two types of point clouds and polygonal meshes and section 5 summarises the main findings of this work.

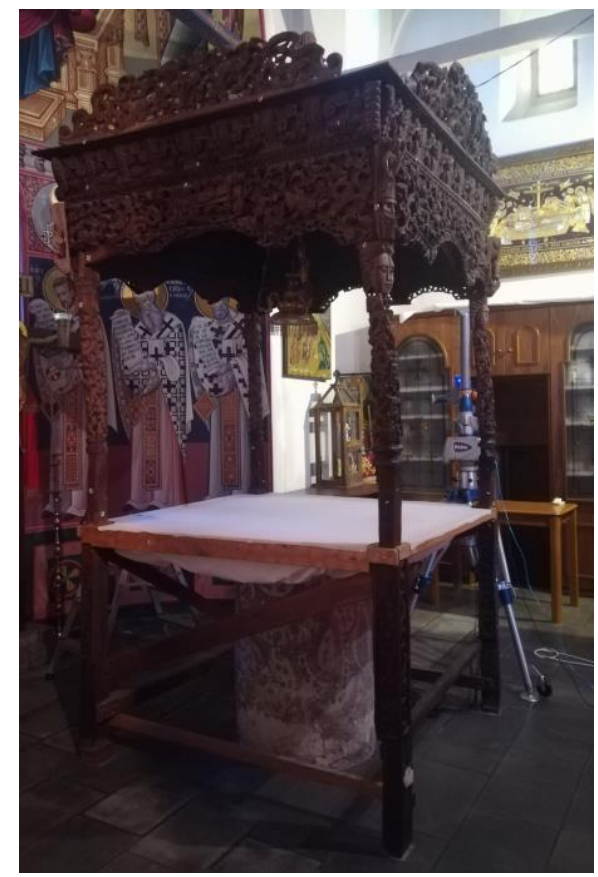

Fig. 1 The ecclesiastical ciborium $\left(17^{\text {th }}\right.$ cent.)

\section{LASER TRACKING PIPELINE}

The pipeline for scanning and virtual restoration processing to form a texture mapped triangle mesh from sensed data is well documented (eg. Bernadini et al., 2002). For RE manufacturing applications, commercial products (e.g. Polyworks, RapidForm) are available to convert point clouds to meshes. These products also include some standard CAE/CAD analysis tools such as measurements on surfaces and comparisons of as-built to originally digitally designed parts.

However, the needs of cultural heritage applications are different from traditional CAE/CAD in several different respects. First, the objects of interest are typically not regularly machined objects, but rather complex freeform shapes that were produced manually and have been worn or broken over time through natural processes. Second, while shape alone is sometimes of interest, the material appearance of the object as well as its shape are of interest. Third, several alteration iterations are needed to evaluate the validity of a change. Whilst in cultural heritage applications, judgments are often made on the basis of appearance and matching images and textual descriptions of appearance, in manufacturing RE it is important to implement more readily quantified requirements such as making parts fit together or minimizing weight. Fourth, the population using digital models in cultural heritage is much 
different from the one using CAE/CAD tools. While cultural heritage specialists may have extensive technical training, their primary interest is in the history of the objects being studied and the people that used them and not in the technology itself. Finally, the hardware and software resources available to cultural heritage projects are generally less complex than those of engineering design projects. In the following, a discussion on the hardware used and the produced products is given.

\subsection{Laser tracking system}

The data acquisition was performed with the FARO ScanArm, which is a Coordinate Measuring Machine (CMM) arm with an attached hand-held laser scanner (Laser Line Probe, LLP) (cf. Fig. 1). The ScanArm's hard probe and the Laser Line Probe can digitize interchangeably without having to remove either component. The FARO LLP works via laser triangulation. The LLP is, at its core, a digital camera that photographs laser light and derives the depth based on the reflection return angle and time. The LLP consists of a pulsing laser emitter that projects a line of laser light, and a charge-coupled device (CCD) sensor, which is a grid of pixels that convert photons of light into electrical charges that are then interpreted as digital images (www.faro.com). The CCD measures the reflection of the laser photons emitted from the probe, and much like a camera, the settings need to account for all of the things that can affect the way that the photons will reflect back to the sensor. The arm has an ergonomic pistol grip to enable the manual measurement of 3D points at any orientation within the arm's spherical reach $(2.8 \mathrm{~m})$, with a precision of $\pm 0.040 \mathrm{~mm}$ and a repeatability of $\pm 0.028 \mathrm{~mm}$. The optical laser system mounted on the arm allows to collect up to 560,000 points per second (www.faro.com).

Prior to a measurement project, a compensation routine procedure (sometimes referred to as calibration) needs to take place. This routine is designed to closely control the position and orientation of the LLP as the user covers multiple quadrants of a compensation plate while moving the LLP. Specifically, the operator takes a series of five strokes covering four positions of the compensation plate while moving the LLP far from and near to the plate. After compensation, it was necessary to set a number of data acquisition settings in the software. Of the data acquisition settings, there are three main variables that convey resolution: the grid size, the maximum point distance (edge length) and the maximum deviation. The grid size setting is used to determine the average distance between points. As the laser line passes across the surface of the material being scanned, the sensor picks up more points than are necessary to create an accurate model. For this project, it was set to $0.17 \mathrm{~mm}$. The maximum edge length setting determines the distance between adjacent laser stripes that will give continuous data. The LLP collects data at a fixed rate, and if the probe is moved too quickly, there will be gaps between the acquired data. The maximum edge length determines how much overlap between the scan lines is acceptable. A small maximum edge length means that if the probe is moved too fast and the overlap is not sufficient, the strips of data that are acquired will be discarded. A larger maximum edge length means that less overlap is necessary to preserve the data, and if there are gaps, they will be filled automatically. This makes the scanning easier, but it also means that the areas where no data points are collected will be filled in with estimated data points, which diminishes the accuracy of the model. The maximum edge length used for this project was $3.5 \mathrm{~mm}$. The maximum deviation setting is a noise reduction tool. Because the CCD sensor works by registering the location of reflected laser light, sometimes photons with the same energy signature as the laser being emitted from the LLP enter the CCD sensor and register as data points. This 'noise' is unavoidable, but it is undesired and therefore it must be accounted for and eliminated if a scan is to meet standards of accuracy for cultural heritage applications. One way to mitigate this is by telling the software that if a data point comes in that deviates from the rest of the data points being collected by a certain distance, it will be discarded as noise. This maximum deviation distance for this project was set to $0.05 \mathrm{~mm}$.

The second group of settings that needed to be defined deal with the way the sensor actually acquires the data. There are four basic settings that can be adjusted: noise threshold, exposure, scan rate, and scan density. Of the four, only two are commonly adjusted to account for the different colour and reflectivity of an object: exposure and noise threshold. Both of these can be automatically obtained by the software, much in the way that automatic settings calculate exposure and the lens aperture for a camera. The noise threshold setting configures the sensitivity of the CCD to the returned photons; the electrical charge of each pixel is measured on a scale of $0-255$, and any value below the noise threshold will be discarded (www.faro.com). For the ciborium, the standard FAROrecommended set point of 15 was used. The exposure setting for the LLP controls the amount of time that the sensor collects light per laser pulse, similar to how the exposure setting controls the length of time for the shutter to be open on a digital camera. The exposure is measured on a scale of 1-80, which corresponds to an exposure time in milliseconds. For lightercoloured objects, a lower exposure setting is needed, and for darker objects, a higher one. If a light object is scanned with too high an exposure, the resulting scan will contain significant noise. If a dark object is scanned with too low an exposure, the electrical charge for each pixel on the CCD will be too low and will be discarded. Because the wood is fairly dark, an exposure setting of 70 was used, which corresponded to 4.375 milliseconds.

Finally, the last two settings are the scan rate and the scan density. These settings control how much information is obtained per laser pulse. The scan rate controls the number of scan lines captured per second. The scan density controls the number of points captured per line. These were left as the default values in the software. The scanning arm has two buttons; the green button, which starts the scanning and the red that pauses it. During the scanning process, it is helpful to pause and restart the scan often. If a scan is done too quickly or the data does not meet the limits of the noise thresholds set prior to scanning, scanned data will be discarded. The data is not discarded on the fly though, it is discarded after the scanning is either paused or stopped. Pausing allows the user to see which parts of the scan are being kept and which parts are being discarded. It is also a good idea to pause the scan prior to repositioning the LLP; if the scanner is still trying to collect data while the LLP is moved, it can scan something not intended to be scanned, or collect noisy data.

Each scan was executed by manually following the surface profile without restriction to a specific angle orientation; in this way, data acquisition was extremely fast. From one position it was not sufficient to acquire the entire object geometry restricted by the arm's length although the arm could be rotated with a large freedom of movement. Therefore, scan overlapping was necessary. The duration of data acquisition was in the order of 5 hours for 8 different set-ups of the instrument. During the 
scanning process, the laser probe has to be kept at a certain distance from the object being scanned. If the probe is too close, the laser cannot return to the sensor window, and likewise if it is too far away. There is a range of distances that work depending on the object being scanned. The LLP has a rangefinder on it to show when the scanner is at the correct distance from the object, and the computer screen will show whether or not data is being collected in real time. Because of the narrow range of distances at which data collection takes place, it is especially important to pay close attention to the screen when scanning the edges of the object at an angle perpendicular to the edge. If the LLP is too far from the edge, the tip of the edge can be in range but the faces not, or if the LLP is held too close, the faces of the object can be in range but the edge not. Since data acquisition is a product of the laser line return to the sensor, it was necessary to scan from a variety of angles to minimize shadow areas where the laser line return was obstructed. Therefore, the most productive data acquisition occurs when the LLP is held perpendicular to the face being scanned, but for the engraved parts more oblique angles were required to capture larger surface.

\subsection{RE digital model}

The digital model reconstruction of the acquired point clouds was performed using the 3D metrology software platform Polyworks V18 by InnovMetric (InnovMetric Software Inc., Quebec, Canada) (Reference Manual 2018). Polyworks is a modularized package that offers a centralized workspace for organizing component parts of $3 \mathrm{D}$ data processing and consists of several modules, each dedicated to a specific phase of the RE procedure, from scan alignment to meshing to inspection. The Inspector module (IMSurvey) allows to scan the objects and align the resulting datasets. The Modeler module covers several essential steps for polygonal models editing, generation of curves, non-uniform rational basis spline (NURBS) patches and models that can be exported as CAD files readable by other software tools. It also allows to compare the acquired data (e.g. point cloud) with a reference (e.g. CAD model), measure the dimensions of specific features and generate comparison and verification reports.

Prior to any processing, registration (or alignment) of the collected scans is essential to be performed. Within the PolyWorks software, the different sets of point clouds are entitled 'Data objects' and can be manually aligned to one or several reference objects by pointing point pairs within each data in the Align menu. An alignment is performed under the Data alignments branch in 'Tree View'. Following the IMSurvey manual guide, the data alignments branch can be described as a group of data alignments that refer to one or multiple Data objects in data alignment groups. In general, data alignment includes the position of point clouds after registration process and also the parameters used and the type of alignment carried out. Each of alignment performed is automatically added to the list of Data alignment group, then it can be changed and compared. The first stage of alignment is performed by selecting at least three common points in the data objects using an initial pairwise ICP (Iterative Closest Point) alignment. The second stage is followed by a global registration step (such as best-fit point-to-surface alignment, best fit cross sections, centre points alignment etc) which improves the final alignment, spreading the errors equally between all view pairs.

After alignment is completed, the point cloud is ready to undertake further processing. In point cloud processing using
PolyWorks there are different operations available to improve them: noise and overlap reduction, redundant points deletion. The main PolyWorks tool for filtering data when the point cloud is too dense is called Subsample. The tool offers random, uniform and curvature-based sampling methods. For this operation the point cloud needs to be visible and selected to perform the procedure. The 'Uniform' method was chosen with a tolerance value based on how much the point cloud needed to be reduced.

The second phase involved the creation of the triangulation mesh, the so-called 'polygon mesh'. Within the software there are three types of data triangulation: Triangulate Data Points, Triangulate Terrain Data Points and Wrap Mesh around Point Cloud. The first two are not suitable for this particular data set and therefore the third algorithm was used. The 'Wrap Mesh around Point Cloud' is probably the best method implemented for creating a mesh because by this way the triangulation is allowed to be best-fitted to a collection of points by subdividing triangles and changing their vertices in $3 \mathrm{D}$ space. The module offers additional options: rejection of outliers, noise reduction and curvature-based subsampling. Fig. 2 shows the created polygonal mesh of the ciborium using the 'Wrap Mesh around Point Cloud' method.

Due to the high complexity of the object (free-form geometry) it was not possible to perform further processing such as curves and patches generation using NURBS construction.

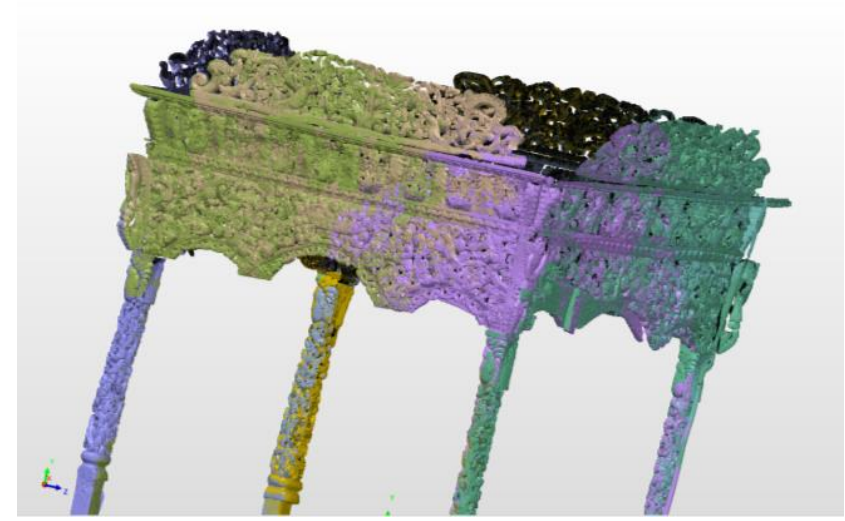

Fig. 2 The 3D point cloud produced by the ScanArm

\section{PHOTOGRAMMETRIC PIPELINE}

The photogrammetric workflow comprised the following steps: Image acquisition with a digital camera;

Camera calibration and image orientation, using a Structure-from Motion (SfM) procedure able to extract homologues points between the images and the unknown camera parameters:

- Dense matching and 3D reconstruction for the generation of dense point clouds;

Polygonal model generation and texture mapping for analyses and visualization.

\subsection{Image acquisition}

The photogrammetric acquisition was carried out in the altar room of the church. A Canon 6D digital single-lens reflex (DSLR) camera which employs a full frame CMOS sensor of $35.8 \times 23.9 \mathrm{~mm}$, with resolution 20.2 effective megapixels and 
$6.5 \mu \mathrm{m}$ pixel size as well as a Canon EF $24 \mathrm{~mm}$ fixed focal length lens providing an f1.4 maximum aperture. A set of 500 images was acquired with imaging acquisition distance of about $40 \mathrm{~cm}$.

Due to dim lights present in the room, external lamps were used in order to avoid specular highlighting that can compromise the photogrammetric process and produce a not faithful texture. Thus, for the purposes of illumination, a Studio Flashlight (1200 Watts) and its control panel was used combined with a soft box of $30 \times 40 \mathrm{~cm}$, for diffused lighting. This was essential in order to achieve both smooth illumination and the use of the smallest aperture value so as the largest depth of field to be provided. The captures were made at 100ASA to ensure the lowest digital noise level at the final produced photos.

A factor that cannot be underestimated during image data acquisition is the availability of sufficient and safe space. The limited space around the ciborium, its reasonable height and the risk of hitting or damaging other assets when moving around with bulky equipment was often stressful.

The acquired images obtained were processed through the image-based 3D modelling software Agisoft PhotoScan. This software is based on the structure-from-motion (SFM) and dense multi-view 3D reconstruction (DMVR) algorithms, and allows to build 3D models by unordered image collections that depict a scene or an object from different viewpoints. First, the software performs the alignment of the images, on the basis of common points in the source photos and matches them to obtain a single point cloud using a scale-invariant feature transform approach. If necessary, the images were manually edited in order to mask the background, leaving only the artefact visible on the pictures. After image alignment, the generation of a dense point cloud was automatically performed by the software using a semi-global dense matching approach. The last step was to generate a photorealistic texture on the $3 \mathrm{D}$ polygonal model. The mesh model was opportunely edited to correct for topological errors and the mesh model was textured with high resolution external texture generated from the original images.

Clearly, the advantage of the SfM procedure is the determination of internal camera geometry, position and orientation automatically. The only cost is the required high degree of overlap in image acquisition to cover the full geometry of the object. However, when the object is complex, as it the case in this work and the images are taken at short distances, even the SfM operator cannot always guarantee the precise orientation of images. An example is given in Fig. 3a which depicts the automatically derived cloud from a façade of the ciborium with problem areas mainly at the top of the object. In order to assist the process, a number of homologous points (tie-points) were manually selected on the images (about 30) which facilitated in the calculation of the rotation and translation parameters. Fig. 3b depicts the derived cloud using manually measured tie-points. The alignment of the images was performed with an accuracy of $0.1 \mathrm{~mm}$. Having the points clouds derived from the two RE systems, the corresponding polygonal surfaces (mesh) were created. Fig. $4 \mathrm{a}$ and $4 \mathrm{~b}$ depict examples of the two different surfaces. The former has an approximate number of $3,000,000$ triangles and the latter has about $22,000,000$ triangles.

In order to compare and later combine the two cloud points for further modelling (derived from SfM and laser scanning), georeferencing was performed in a common coordinate system.
This was accomplished by ten points located on the four sides of the ciborium and measured using the LLP at the quoted precision of the manufacturer.

The final stage refered to the creation of the textured models by providing external texture to the final mesh models and thus the creation of the orthoimage. In the first case, the orthoimage was derived using the SfM mesh (Fig. 5a) and in the second case the orthoimage was derived using the laser scanning mesh (Fig. 5b). The visual inspection of the two orthoimages indicates that the SfM-derived one presents a complete model but the laserderived one has sharper details despite the occasional gaps.

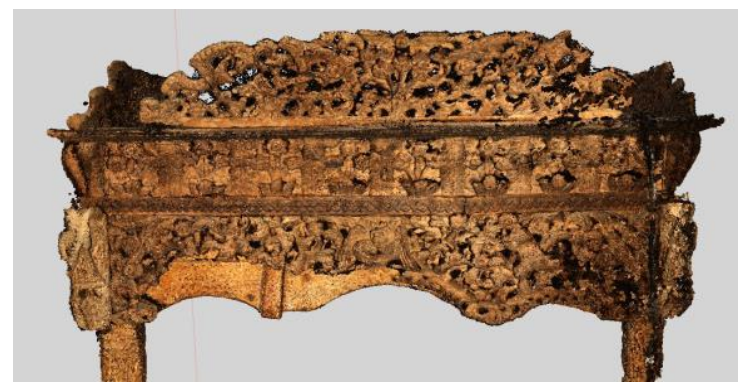

Fig. 3a Point cloud from automatic SfM procedure without manually introduced tie points

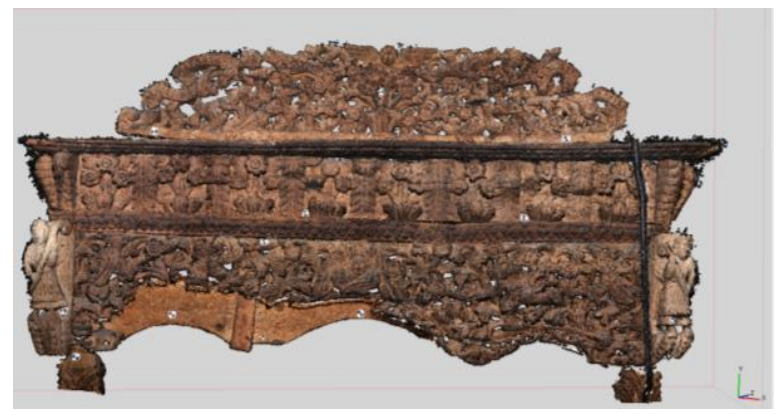

Fig. 3b Correct point cloud produced using SfM procedure with manually measured tie points (about 4.000 .000 points)

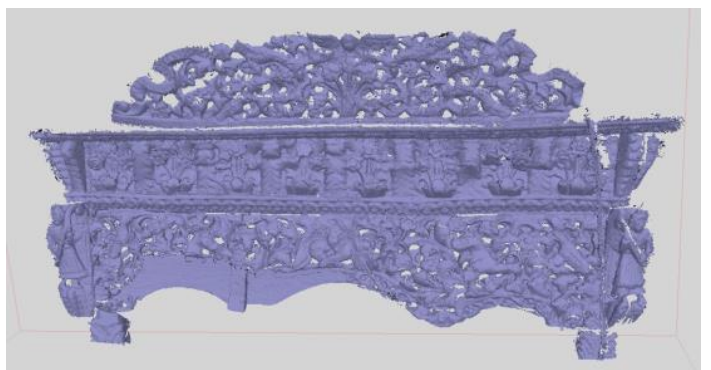

Fig. 4a Polygonal surface derived from the SfM procedure

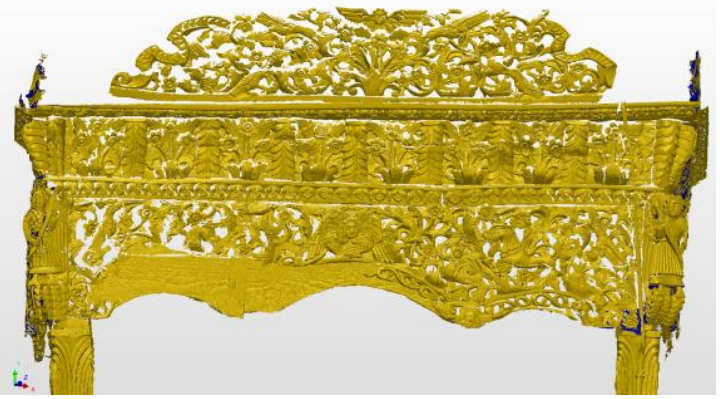

Fig. 4b Polygonal surface derived from the scanning procedure 


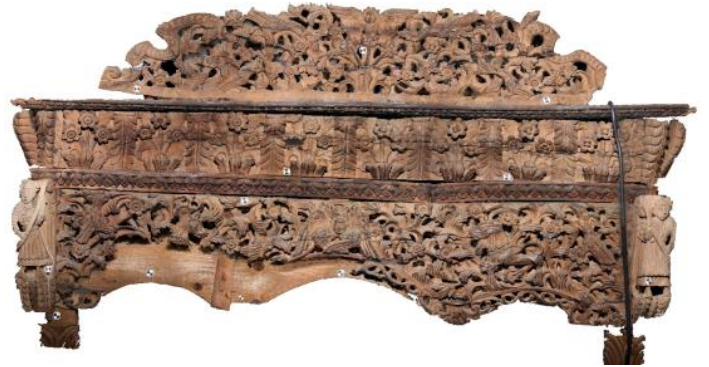

Fig. 5a Orthoimage derived using the DSM from the SfM

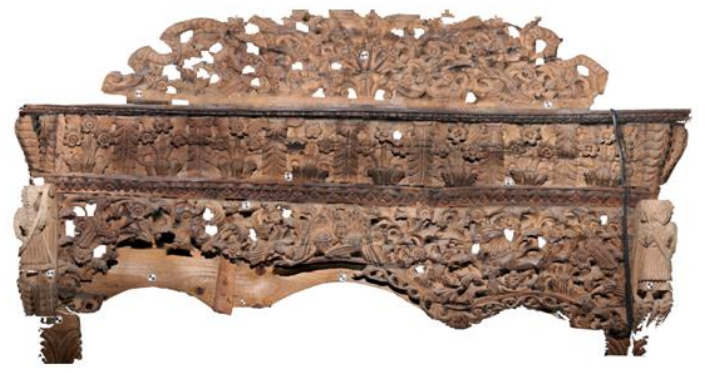

Fig. 5b Orthoimage derived using the DSM from scanning

\section{RESULTS COMPARISON AND DICSUSSION}

A number of comparisons were performed between the results obtained with the two diverse RE systems: the first referred to comparing the two point clouds, and the second referred to the polygonal models generated from the point clouds. All the comparisons were carried out considering the results obtained with the laser arm system as 'standard reference'. The differences were computed via a shortest distance algorithm between the two results and were represented as a coloured map. For the comparisons, the CloudCompare open source software was used.

For the point-to-point comparison, the two clouds (SfM and laser scanning) were given as input in order to calculate their difference. The coloured map of Fig. 6a depicts the maximum distance, highlighted in colour blue, between the two types of clouds with a mean of $1.28 \mathrm{~mm}$ and standard deviation $\pm 3.24 \mathrm{~mm}$. The comparison between the polygonal models ( $\mathrm{SfM}$ derived and laser scanning derived) is shown in the coloured map of Fig. $6 \mathrm{~b}$ with the maximum distance highlighted in colour purple between the two types of surfaces with a mean of $1.33 \mathrm{~mm}$ and standard deviation $\pm 1.95 \mathrm{~mm}$. The mesh-to-mesh volume comparison indicates differences of $2 \mathrm{~mm}$ for more than $50 \%$ of matching cells (Fig. 6c).

From the comparisons, it can be noticed that the two models obtained with the two diverse RE system are substantially similar. The divergence between the two RE models is very low but the real difference is given by the definition of the details. For example, the same portion of the two polygonal models, which is at around the centre part of the object, is shown to visualize the high details definition. At the sides of the object the differences are larger mainly from the SfM derived mesh. The laser derived mesh presents a uniform precision.

In Table 1, a summary between the products derived from the two RE systems (laser tracking and Digital Close-range
Photogrammetry -DCRP) is reported in terms of number of acquired points, number of triangles, data acquisition time, post-processing time, and RE system costs. The photogrammetric system is of very low cost and faster than the laser arm system, but the latter is more precise giving greater details although with larger gaps due to the way the system is operating (triangulation principle).

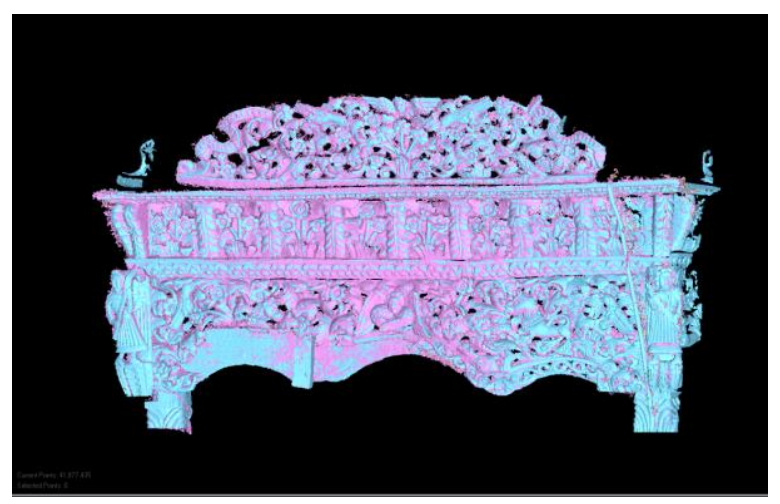

Fig. 6a Point-to-point comparison between the SfM and laser clouds

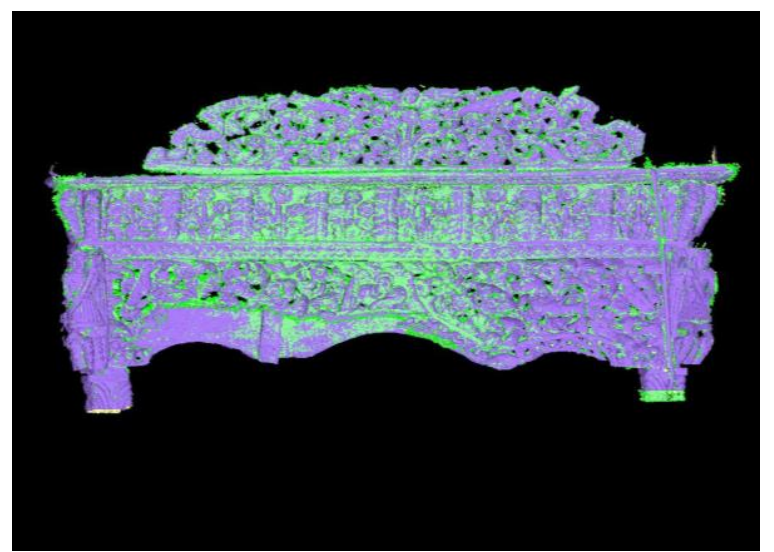

Fig. 6b Mesh-to-mesh comparison between the SfM and laser surfaces

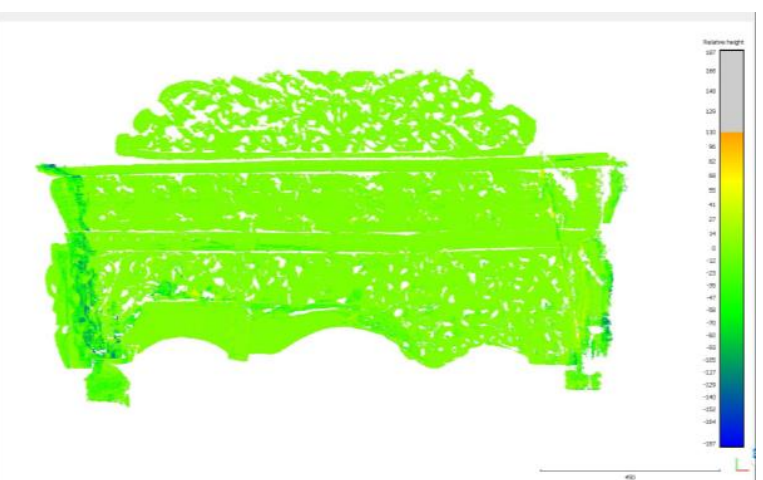

Fig. 6c Mesh-to-mesh volume comparison between the SfM and laser derived surfaces

Table 1. Comparison of the two RE models

\begin{tabular}{|l|l|l|}
\hline & Laser tracking & DCRP \\
\hline No of points & $30,000,000$ & $4,000,000$ \\
\hline No of triangles & $42,000,000$ & $5,500,000$ \\
\hline Acquisition time & $5 \mathrm{hr}$ & $2 \mathrm{hr}$ \\
\hline Post-processing time & 1 day & 7 days \\
\hline Cost & Very high & Low \\
\hline
\end{tabular}




\section{CONCLUDING REMARKS}

A case study of laser-based and multi image-based RE procedures applied to a tangible cultural heritage artefact has been illustrated by examining all the steps required to convert the complex free-form geometry of a wooden engraved ecclesiastical ciborium into its 3D digital representation.

On the basis of hardware features considerations, the laser armbased RE procedure, as expected, proved to be the most precise but the produced cloud has larger number of gaps due to the way the system operates (triangulation principle). Also, the point cloud acquisition time was almost 2.5 times longer than the phtogrammetric-based RE procedure. The digital photogrammetric procedure is a mature technology and less time consuming in the data acquisition stage.

The analysis of the obtained results takes into consideration no only the accuracy of the final 3D model of the freeform object but also the required time to achieve this and the investment relevance for system purchase. The point clouds derived from the two systems are substantially different in terms of number of points with the laser arm producing volumes of seven times more than the photogrammetric process. The 3D digital models of the object obtained with the two RE systems, were comparatively evaluated via coloured maps defining the mean distance between the two results. By this comparison, the 3D polygonal surfaces derived from the two methods appear to be similar with an overall mean distance of $1.33 \mathrm{~mm}$. The derived orthoimage using as DSM the laser point cloud has sharper details but with larger number of gaps when compared to the respective orthoimage produced using the SfM derived point cloud.

Clearly, the investment cost for the laser arm-based system is much higher than that for digital cameras. However, in view of future developments, the availability of a precise 3D digital model derived from both the two techniques as examined in this work, could offer several opportunities within the cultural heritage as well as the manufacturing fields.

\section{ACKNOWLEDGEMENTS}

The Monastery of Agios Stefanos in Meteora, Greece is gratefully acknowledged for their permission and valuable help.

\section{REFERENCES}

Bernard, A., Laroche, F., Ammar-Khodja, S. Perry, N., 2007. Impact of New 3D Numerical Devices and Environments on Redesign and Valorisation of Mechanical Systems. CIRP Annals - Manufacturing Technology 56 (1): 143-148.

Bernardini, F., and Rushmeier, H., 2002.The 3D Model Acquisition Pipeline. Comput. Graph. Forum, 21_2_, pp. 149172.

Clini P., Frapiccini, N., Mengoni, M., Nespeca, R., Ruggeri, L., 2014. SFM technique and focus stacking for digital documentation of archaeological artifacts. Proc. ISPRS Volume XLI-B5, 2016 XXIII ISPRS Congress, 12-19 July 2016, Prague, Czech Republic.

Gallo, G., Muzzupappa, M., Bruno, F., 2014. 3D reconstruction of small sized objects from a sequence of multi-focused images.
Journal of Cultural Heritage, Vol. 15(2), pp. 173-182.

Guidi, G., Rodríguez Navarro, O., Gonizzi-Barsanti, S., Micoli, L.L., Russo, M., 2013. Quick textured mesh generation in Cultural heritage digitization. Proc. Built Heritage 2013 Conference - Monitoring Conservation and Management, pp.874-882.

Martin, J., 2017. The real-time promise of 7DoF laser tracking. Quality Magazime, March, 2017(www.qualitymag.com).

Nicolae, C., Nocerino, E., Menna, F., Remondino, F., 2014. Photogrammetry applied to problematic artefacts. Proc. ISPRS Technical Commission V Symposium, Volume XL-5, 201423 25 June, Riva del Garda, Italy.

Pruit, J.B., Clement, N.G., Tapanila, L., 2017. Laser and structured light scanning to acquire 3D morphology. Virtual Paleontology 22:57-69 https://doi.org/10.1017/scs.2017.8

Raja, V., and Fernandes K. J., eds. 2008. Reverse Engineering: An Industrial Perspective. 242 p. London: Springer-Verlag.

Segreto, T., Caggiano, A. Teti, R., 2011. Diverse Non Contact Reverse Engineering Systems for Cultural Heritage Preservation. In 7th Int. Conf. on Digital Enterprise Technology - DET 2011, 486-493, Sept. 28-30, Patras, ISBN 978-96088104-2-6.

Segreto, T., Bottillo, A., Teti, R., Galantucci, L.M., Lavecchia, F., Galantucci, MB, 2016. Non-contact reverse engineering modeling for additive manufacturing of down scaled cultural artefact. In 10th CIRP Conf. on Intelligent Computation in Manufacturing Engineering - CIRP ICME '16, 20-22 July, Naples, Italy.

Schaich, M. 2013.Combined 3D scanning and photogrammetry surveys with 3D database support for archaeology \& cultural heritage. A practice report on ArcTron's information system aSPECT3D. Proc. Photogrammetric Week 2013, D. Fritsch (ed.). Wichmann, Berlin/Offenbach, pp. 233-246. 